\title{
Effect of Overlaid Pavement on Transverse Load Distribution in Existing Assembly Slab Bridges
}

\author{
G.H. Wang ${ }^{1,2}$, X.Y. Liu ${ }^{1, *}$, C.L. Wei ${ }^{1}$, L. Shen ${ }^{1,3}$ and T.X. Jiang ${ }^{1}$ \\ ${ }^{1}$ School of Civil Engineering and Architecture, Changsha University of Science and Technology, Changsha, 410076, China; \\ ${ }^{2}$ The College of Civil Engineering and Architecture, Hunan Institute of Science and Technology, Yueyang, 414000, China; \\ ${ }^{3}$ Department of Civil and Environmental Engineering, Louisiana State University, Baton Rouge, LA 70803, USA
}

Received 19 August 2016; Accepted 15 December 2016

\begin{abstract}
The bearing capacity of old assembly slab bridges is reduced by component cracking, material aging, and structural damage. To avoid excessive dismantling of bridges with inadequate bearing capacity, the influence mechanism of overlaid pavement on existing assembly slab bridges was analyzed by theoretical analysis and numerical simulation in this study. The basic equation for assembly slab bridges was established by considering the bending resistance and shearing resistance of the overlaid pavement and based on the fundamental principle of force method. The formula for the vertical coordinate of the influence line of the transverse load distribution (TLD) was constructed using a half-sine unit load. On this basis, the variations in the vertical coordinate with rigidity, slab width, and bridge span were analyzed. Moreover, the influences of the overlaid layer thickness on assembly slab bridges with different spans (13, 16, and $20 \mathrm{~m})$ were analyzed. Results show that the overlaid pavement can significantly affect the influence line of the TLD. The influence of the overlaid pavement on the TLD factor should be taken into account for assembly slab bridges with spans of $16 \mathrm{~m}$ or smaller. However, such influence is negligible for bridges with spans of $20 \mathrm{~m}$ or larger. The overlaid pavement can increase structural integrity and reduce the TLD factor to some extent. But an overlaid pavement thickness of more than $20 \mathrm{~cm}$ only slightly influences the TLD factor. The study can meet the demand of reconstruction of old bridges based on overlaid pavement. The proposed method solves issues related to the low bearing capacity of these old assembly slab bridges.
\end{abstract}

Keywords: Bridge engineering, Assembly slab bridges, Bridge deck pavement, Transverse load distribution factor

\section{Introduction}

China has achieved rapid economic development in recent years. The load-bearing capacity of existing bridges should be increased to cope with the continuous increase in number of heavy-duty and overloaded vehicles. Particularly, assembly slab bridges in low-grade highways cannot meet such high bearing capacity [3]. The limitations of existing bridges in China are attributed to the low load design standards, poor construction quality control because of limited construction budget, and component cracking [1], material aging [2], and structural damage caused by improper operating maintenance and management. However, dismantling and reconstructing old bridges not only waste construction resources and disturb the ecological environment, but also cause long-term traffic interruption [4]. Thus, reinforcement is the best way to solve the problems related to the low bearing capacity of these assembly slab bridges. Nevertheless, with limited maintenance budget, these bridges are often reinforced with a layer of 15-30 cm-thick reinforced concrete pavement in accordance with engineering experiences. This

E-mail address: wgh325@126.com

ISSN: 1791-2377 @ 2017 Eastern Macedonia and Thrace Institute of Technology. All rights reserved. reinforcement can increase structural integrity and disperse loads to some extent, thus realizing the goal of increasing the bearing capacity of the bridge girder. However, further research on the appropriate thickness of overlaid pavement and its degree of influence on the stress status of girder is necessary. Therefore, an assembly slab bridge reinforcement model should be established urgently with consideration to the reinforced concrete pavement to optimize the thickness of overlaid pavement and enhance the reinforcement effect.

Stresses on the girder of assembly slab bridges reinforced by overlaid pavement were analyzed theoretically in this study. A stress analysis of the assembly slab bridge before and after reinforcement with overlaid pavement was conducted based on the principle of equilibrium of forces. An analytical model that considers the bending resistance and shearing resistance of overlaid pavement was established using the basic equation of force method.

\section{State of the art}

It is common to pour an $8-12 \mathrm{~cm}$-thick reinforced concrete pavement over the girder of assembly hollow slab bridges during deck construction. In bridge reinforcement by overlaid pavement [5],[6], some portions of the old concrete 
pavement have to be removed until the internal reinforcing steel bars are exposed to wedge the reinforcing steel bars in the new concrete pavement into an integral whole with the reinforcing steel bars in the original pavement. This process ensures the normal cementation of the new concrete pavement and the old concrete pavement and enhances the structural integrity and stress uniformity in the precast slabs [7]. The influences of the pavement on girder rigidity are often overlooked, and the pavement is only viewed as a component layer when calculating the stresses on the bridge. In reality, however, the reinforced concrete pavement forms a composite structure with the girder, and they bear the stresses together. The independent overlaid pavement is a rigid structural layer with both bending resistance and shearing resistance. Most of existing studies on overlaid pavement mainly have focused on the mechanical analysis, design theory, material properties, and disease prevention [915], but only few have analyzed the stresses shared by the overlaid pavement and girder. The reinforcement of bridge structures with small and medium spans by overlaid pavement was introduced from the perspective of applicable conditions, material requirements, mechanical characteristics, design calculation, construction measures, and process quality control [7]. In a previous study [16], destruction tests were conducted on bridge structures with full-size hollow slab pavement and without pavement. The influences of the overlaid pavement on cracking behavior, deformation, and bearing capacity girder were analyzed, and its effect on the overall mechanical performance of the bridge structure was explored by conducting a load test. $\mathrm{Lu}$ et al. [17] used the hinged plate method to calculate the transverse load distribution (TLD) factors of 13, 16, and 20 $\mathrm{m}$ hollow slab bridge structures. They examined the influences of the overlaid pavement using rigidity $\gamma$ and found that the overlaid pavement slightly influences the TLD factor. Liu et al. calculated the modal flexibility by a modal parameter method, and the deflections of the midspans of different beams were extracted from the modal flexibility to determine the TLD factor [18]. Wang et al. established equations for the influence line of the TLD in a hinged plate bridge structure, which facilitated the design and calculation of a hinged plate bridge structure with different section forms and different quantities of plate girders [19]. $\mathrm{Wu}$ and Zhang [20] explored the effects of overlaid pavement on the mechanical performance of a simply supported hollow slab bridge by finite-element calculation and static test and found that the overlaid pavement could effectively reduce the responses of the bridge.

In summary, the existing stress calculation for an assembly slab bridge mainly includes lookup-table calculation in the hinged plate method [21]. The hinged plate method considers overlaid pavement by correcting the rigidity $(\gamma)$ of the bridge deck pavement. Actually, it only considers the shearing resistance of the overlaid pavement, but neglects its bending resistance.

In the current study, a calculation model which involves both bending resistance and shearing resistance of overlaid pavement was established using the basic principle of force method. By this method, the influences of the overlaid pavement on the TLD in the assembly slab bridge and, consequently, on the mechanical performance of the bridge were identified.

The rest of this study is organized as follows: Section 3 discusses the stress analysis of the assembly slab bridge reinforced with overlaid pavement and the establishment of the mechanical calculation model involving the bending resistance and shearing resistance of the overlaid pavement. Section 4 analyzes the effects of the overlaid pavement on the TLD factors of assembly slab bridges with different spans and slab widths. Section 5 presents the conclusions.

\section{Methodology}

3.1 Stress analysis of the assembly slab bridge with consideration for the overlaid pavement

At present, the hinged plate method is generally applied to calculate the TLD factor of an assembly hollow slab bridge. In the hinged plate method, the joint between the plates only transmits the shearing force, but the transmission of the bending moment and normal force is neglected. For an assembly slab bridge with inadequate bearing capacity, the reinforced concrete pavement is a rigid structural layer that can transmit both bending moment and shearing force. Thus, the calculated results obtained by the hinged plate method cannot reflect the actual stress conditions of bridge structures. In this study, the influence line of the TLD was calculated by considering the bending resistance and shearing resistance of the reinforced concrete pavement. The bridge considered was a simply supported girder bridge consisting of five hollow slabs (Fig. 1.(a)).

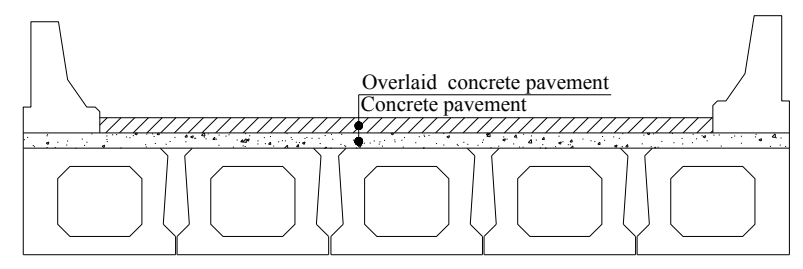

(a) Cross section of the bridge structure

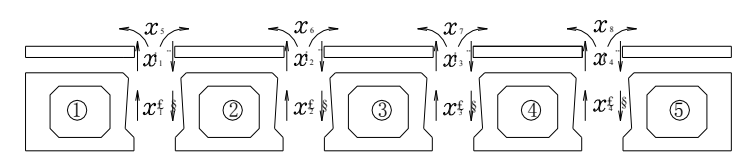

(b) Calculation of section stresses

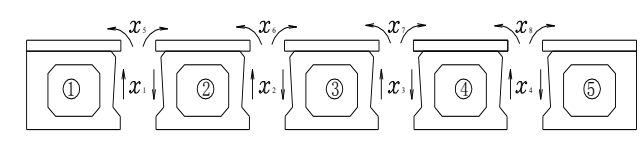

(c) Calculation of section stresses

Fig. 1. Stress analysis of an assembly hollow slab bridge

On the assumption that a half-sine unit load $p(x)=1 \times \sin \left(\frac{\pi x}{l}\right)$ is applied to the slab \#1 and the reinforced concrete pavement is disconnected at the joint $(x=0: l)$, the joint between the hollow slabs only transmits a vertical shearing force, whereas the reinforced concrete pavement transmits a shearing force and a bending moment. The shearing forces between the hollow slabs are $x_{1}^{\prime}, x_{2}^{\prime}, x_{3}^{\prime}$, and $x_{4}^{\prime}$ (Fig. 1.(b)). The shearing forces between the pavement sections are $x_{1}^{\prime \prime}, x_{2}^{\prime \prime}, x_{3}^{\prime \prime}$, and $x_{4}^{\prime \prime}$, and the bending moments between the pavement sections are $x_{5}, x_{6}, x_{7}$, and $x_{8}$ (Fig. 1.(b)). For the convenience of analysis, the shearing forces were integrated (Fig. 1(c)) into $x_{1}, x_{2}, x_{3}$, and $x_{4}$, where $x_{i}=x_{i}{ }^{\prime}+x_{i}{ }^{\prime \prime}(i=1,2,3,4)$. 


\subsection{Calculation model}

According to the principle of the force method, the basic equation is:

$\left[\delta_{i j}\right]\left\{x_{i}\right\}+\left\{\delta_{i p}\right\}=0(i$ or $j=1,2,3 \mathrm{~L} 8)$

where $\delta_{i j}$ is the $i^{\text {th }}$ relative deflection caused by the unit force $x_{j}=1$ from $j(i=j$ and $i \neq j), \delta_{i p}$ is the $i^{\text {th }}$ relative deflection caused by the external loads, and $x_{i}$ is the peak of the $i^{\text {th }}$ redundant force element.

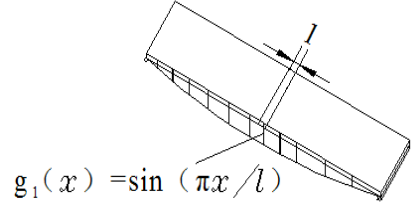

(a)

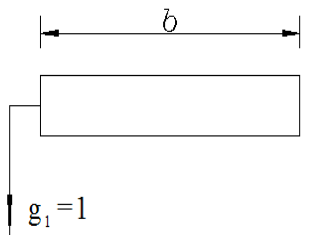

(b)

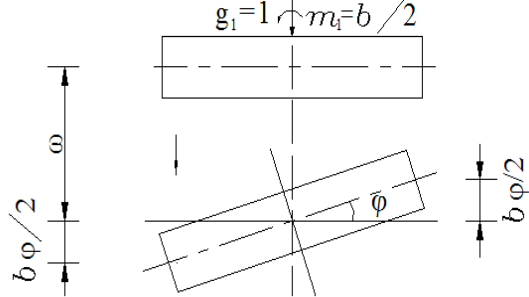

(c)

Fig. 2. Stress analysis under the effect of redundant shearing force

Let $\varphi^{\prime}$ in the coefficient be the girder's torsion angle caused by the unit bending moment at the joint.

$$
\frac{m_{T}}{\varphi}=\frac{x_{i}}{\varphi^{\prime}} \varphi^{\prime}=\frac{\varphi x_{i}}{m_{T}}=\frac{\varphi}{b / 2}=\frac{2 \varphi}{b}
$$

Therefore, the coefficient matrix $\left[\delta_{i j}\right]$ is:

(a)

(b)

Fig. 3. Stress analysis under the effect of redundant bending moment

$$
\left[\delta_{i j}\right]=\left[\begin{array}{cccccccc}
2\left(\omega+\frac{b}{2} \varphi\right) & -\left(\omega-\frac{b}{2} \varphi\right) & 0 & 0 & 0 & \varphi^{\prime} \frac{b}{2} & 0 & 0 \\
-\left(\omega-\frac{b}{2} \varphi\right) & 2\left(\omega+\frac{b}{2} \varphi\right) & -\left(\omega-\frac{b}{2} \varphi\right) & 0 & -\varphi^{\prime} \frac{b}{2} & 0 & \varphi^{\prime} \frac{b}{2} & 0 \\
0 & -\left(\omega-\frac{b}{2} \varphi\right) & 2\left(\omega+\frac{b}{2} \varphi\right) & -\left(\omega-\frac{b}{2} \varphi\right) & 0 & -\varphi^{\prime} \frac{b}{2} & 0 & \varphi^{\prime} \frac{b}{2} \\
0 & 0 & -\left(\omega-\frac{b}{2} \varphi\right) & 2\left(\omega+\frac{b}{2} \varphi\right) & 0 & 0 & -\varphi^{\prime} \frac{b}{2} & 0 \\
0 & -\varphi^{\prime} \frac{b}{2} & 0 & 0 & 2 \varphi^{\prime} & -\varphi^{\prime} & 0 & 0 \\
\varphi^{\prime} \frac{b}{2} & 0 & -\varphi^{\prime} \frac{b}{2} & 0 & -\varphi^{\prime} & 2 \varphi^{\prime} & -\varphi^{\prime} & 0 \\
0 & \varphi^{\prime} \frac{b}{2} & 0 & -\varphi^{\prime} \frac{b}{2} & 0 & -\varphi^{\prime} & 2 \varphi^{\prime} & -\varphi^{\prime} \\
0 & 0 & \varphi^{\prime} \frac{b}{2} & 0 & 0 & 0 & -\varphi^{\prime} & 2 \varphi^{\prime}
\end{array}\right]
$$

and the equation for redundant force is: 


$$
\left[\begin{array}{cccccccc}
2(1+\gamma) & \gamma-1 & 0 & 0 & 0 & \gamma & 0 & 0 \\
\gamma-1 & 2(1+\gamma) & \gamma-1 & 0 & -\gamma & 0 & \gamma & 0 \\
0 & \gamma-1 & 2(1+\gamma) & \gamma-1 & 0 & -\gamma & 0 & \gamma \\
0 & 0 & \gamma-1 & 2(1+\gamma) & 0 & 0 & -\gamma & 0 \\
0 & -\gamma & 0 & 0 & 2 \gamma & -\gamma & 0 & 0 \\
\gamma & 0 & -\gamma & 0 & -\gamma & 2 \gamma & -\gamma & 0 \\
0 & \gamma & 0 & -\gamma & 0 & -\gamma & 2 \gamma & -\gamma \\
0 & 0 & \gamma & 0 & 0 & 0 & -\gamma & 2 \gamma
\end{array}\right]\left\{\begin{array}{c}
x_{1} \\
x_{2} \\
x_{3} \\
x_{4} \\
\frac{2}{b} x_{5} \\
\frac{2}{b} x_{6} \\
\frac{2}{b} x_{7} \\
\frac{2}{b} x_{8}
\end{array}\right\}+\left\{\begin{array}{l}
-1 \\
0 \\
0 \\
0 \\
0 \\
0 \\
0
\end{array}\right\}=0
$$

where $\gamma$ is the rigidity, with $\gamma=5.8 \frac{I}{I_{T}}\left(\frac{b}{l}\right)^{2}$. Then, the above equation can be solved:

$$
\left\{\begin{array}{l}
x_{1}=\frac{4(1+4 \gamma)}{5(1+8 \gamma)} \\
x_{2}=\frac{3}{5(1+8 \gamma)} \\
x_{3}=-\frac{2(-1+4 \gamma)}{5(1+8 \gamma)} \\
x_{4}=\frac{1-8 \gamma}{5(1+8 \gamma)} \\
x_{5}=-\frac{8 \gamma b}{5(1+8 \gamma)} \\
x_{6}=-\frac{(3+32 \gamma) b}{10(1+8 \gamma)} \\
x_{7}=-\frac{2(1+6 \gamma) b}{5(1+8 \gamma)} \\
x_{8}=-\frac{(3+8 \gamma) b}{10(1+8 \gamma)}
\end{array}\right.
$$

The vertical coordinate of the influence line of the TLD in slab $\# 1$ can be calculated using solutions $x_{i}(i=1,2,3,4)$; consequently, the TLD factor can be calculated. The vertical coordinates of the influence lines of the other slabs can be calculated following the same process. The vertical coordinate of the influence line of slab \#1 can be expressed as:

$$
\left\{\begin{array}{l}
\eta_{11}=1-x_{1} \\
\eta_{12}=x_{1}-x_{2} \\
\eta_{13}=x_{2}-x_{3} \\
\eta_{14}=x_{3}-x_{4} \\
\eta_{15}=x_{4}
\end{array}\right.
$$

\section{Result analysis and discussion}

4.1 Relationship between the vertical coordinate of the influence line of the TLD and $\gamma$

The vertical coordinates of the influence lines of the shearing resistance and bending resistance of the reinforced concrete pavement are considered in Eq. (5). The hollow- slab calculation equation that neglects the overlaid pavement is given in [5]. The solutions to this equation are

$$
\left\{\begin{array}{l}
x_{1}^{\prime}=\frac{4\left(1+7 \gamma+7 \gamma^{2}+\gamma^{3}\right)}{5+60 \gamma+126 \gamma^{2}+60 \gamma^{3}+5 \gamma^{4}} \\
x_{2}^{\prime}=\frac{(1-\gamma)\left(3+10 \gamma+3 \gamma^{2}\right)}{5+60 \gamma+126 \gamma^{2}+60 \gamma^{3}+5 \gamma^{4}} \\
x_{3}^{\prime}=\frac{2(1+\gamma)\left(1-2 \gamma+\gamma^{2}\right)}{5+60 \gamma+126 \gamma^{2}+60 \gamma^{3}+5 \gamma^{4}} \\
x_{4}^{\prime}=-\frac{(-1+\gamma)^{3}}{5+60 \gamma+126 \gamma^{2}+60 \gamma^{3}+5 \gamma^{4}}
\end{array}\right.
$$

Similarly, the vertical coordinates $\left(\eta_{11}^{\prime}, \eta_{12}^{\prime}, \eta_{13}^{\prime}, \eta_{14}^{\prime}\right.$, and $\eta_{15}^{\prime}$ ) of the influence lines can be calculated. Thus, the influences of the reinforced concrete pavement on the stresses of the hollow slab can be analyzed using the equation $\Delta \eta_{1 i}=\eta_{1 i}-\eta_{1 i}^{\prime}(i=1,2,3,4,5)$. The calculation table of the TLD factor based on the hinged plate method is given in [5]. The rigidity $(\gamma)$ ranges between 0.0 and 2.0. The $\Delta \eta_{1 i} / \eta_{1 i}$ curves are generated (Fig. 4), where $\gamma$ ranges between 0.0 and 2.0 and is used to analyze the influences of the overlaid pavement on the TLD factor.

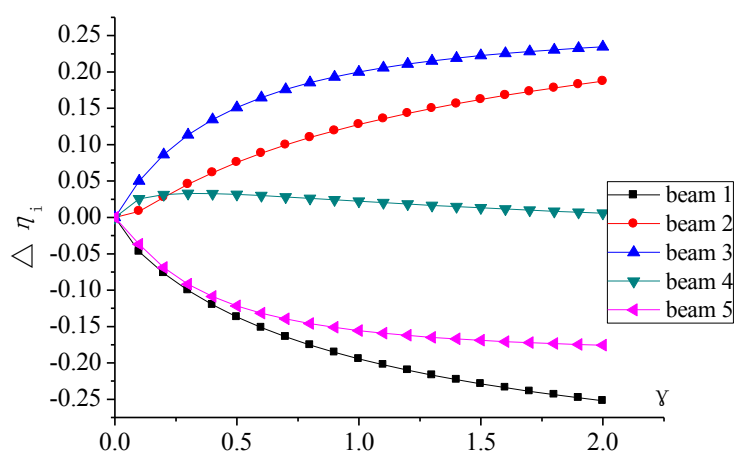

(a) Relation curve between $\Delta \eta_{1 i}$ and $\gamma$ 


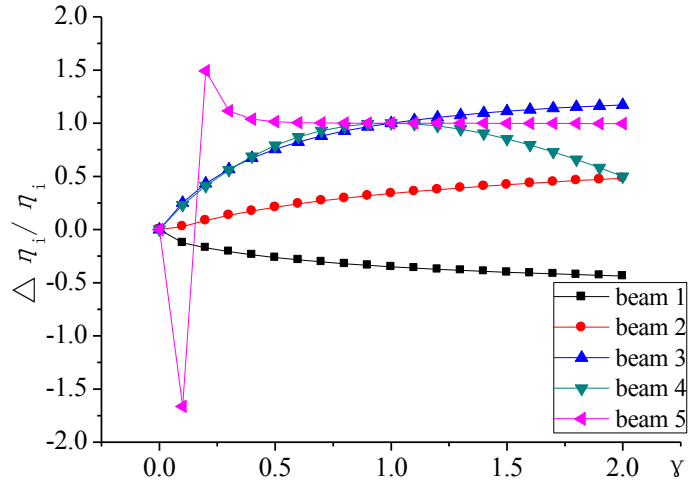

(b) Relation curve between $\Delta \eta_{1 i} / \eta_{1 i}$ and $\gamma$

Fig. 4. Variation curves of $\Delta \eta_{1 i}$ and $\Delta \eta_{1 i} / \eta_{1 i}$ with respect to $\gamma$

Fig. 4. shows that: (1) $\Delta \eta_{1 i}$ changes significantly with an increase in $\gamma$, particularly over the $\gamma$ range of $0-0.5$. (2) With an increase in $\gamma, \Delta \eta_{11}$ and $\Delta \eta_{15}$ continuously decrease, $\Delta \eta_{12}$ and $\Delta \eta_{13}$ gradually increase, and $\Delta \eta_{14}$ slightly changes $(<0.035)$. (3) With an increase in $\gamma$, $\Delta \eta_{1 i} / \eta_{1 i}$ remarkably changes (maximum of $150 \%$ ). These results show that the overlaid pavement can significantly influence the TLD.

4.2 Relationship of the vertical coordinate of the influence line with slab width and span

The rigidity coefficients $\left(\gamma_{13}, \gamma_{16}\right.$, and $\left.\gamma_{20}\right)$ of the three assembly hollow slab bridge structures with spans of 13,16 , and $20 \mathrm{~m}$ are calculated (Tab. 1.).

Table 1. Values of $\gamma$ for different spans and slab widths

\begin{tabular}{c|c|c|c}
\hline $\begin{array}{c}\text { Slab } \\
\text { Width }(\mathrm{m})\end{array}$ & 13 & 16 & 20 \\
\hline 1 & 0.018 & 0.014 & 0.01 \\
1.25 & 0.024302 & 0.01754 & 0.012791 \\
\hline
\end{tabular}

Fig. 5. shows that: (1) the $\Delta \eta_{1 i}$ values for different spans present the same variation law: initial increase and subsequent decrease. (2) The influence lines of the TLD for different slab widths present the same variation law. (3) A larger span results in a smaller difference between the vertical coordinates of the influence line as well as better stress uniformity.

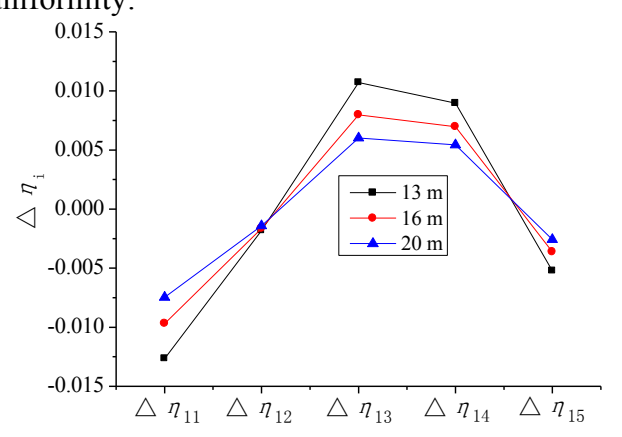

(a) Comparison of $\Delta \eta_{1 i}$ values when slab width is $1 \mathrm{~m}$



(b) Comparison of $\Delta \eta_{1 i}$ values when slab width is $1.25 \mathrm{~m}$

Fig. 5. Comparison of $\Delta \eta_{1 i}$ values for different slab widths and spans

\subsection{Comparison of three pavement-processing methods}

The TLD factors of the three assembly hollow slab bridge structures with different spans $(13,16$, and $20 \mathrm{~m})$ are calculated. In the calculation, the deck width is $12.0 \mathrm{~m}(0.5$ $\mathrm{m}$ [crash barrier] $+11 \mathrm{~m}$ [roadway] $+0.5 \mathrm{~m}$ [crash barrier] $=$ $12.0 \mathrm{~m}$ ), the middle slab is $1.25 \mathrm{~m}$ wide, the reinforced concrete pavement is $10 \mathrm{~cm}$ thick, and the asphalt concrete pavement is $10 \mathrm{~cm}$ thick (Fig. 6).



Fig. 6. Cross-sectional drawing (unit: m)

To further compare the TLD factors, three calculation methods are applied in this study: (1) the proposed method that considers the bending resistance and shearing resistance of the pavement, (2) the hinged plate method that disregards the reinforced concrete pavement when calculating rigidity $\gamma$, and (3) the hinged plate method that considers the reinforced concrete pavement when calculating rigidity $\gamma$. The calculated TLD factors of the three bridge structures by these three methods are shown in Fig. 7.

Fig. 7. shows that (1) the results of the proposed method are smaller than the results of the hinged plate method that disregards the overlaid pavement, but the TLD factor of the boundary beam is consistently higher in the proposed method than those in the other two methods. (2) The maximum TLD factor of the slab decreases with an increase in the span. The calculated results of the three methods are similar with each other. Therefore, the hinged plate method can be directly used to calculate the TLD factors of the bridge structures with large spans. However, the TLD factors of the bridge structures with small spans $(\leq 16 \mathrm{~m})$ should be calculated by the hinged plate method that considers the overlaid pavement. (3) The degrees of stress uniformity in all slabs are better when the bending resistance and shearing resistance of the overlaid pavement are considered.

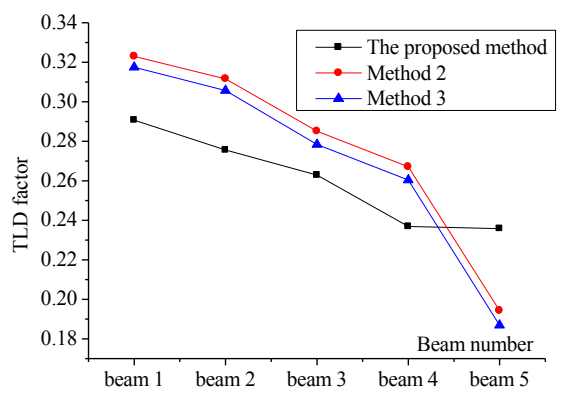


(a) TLD factor of the $13 \mathrm{~m}$ bridge

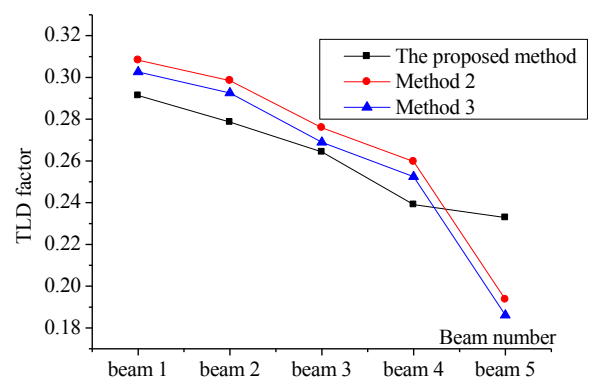

(b) TLD factor of the $16 \mathrm{~m}$ bridge

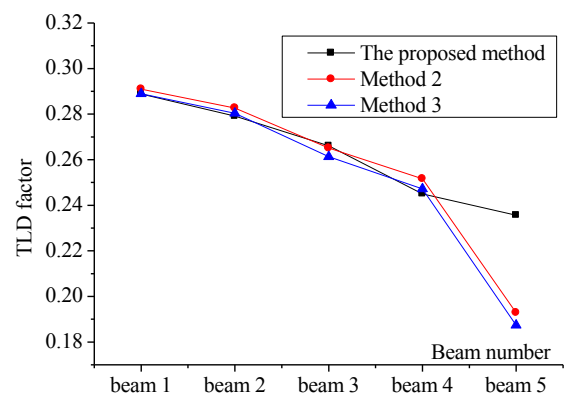

(c) TLD factor of the $20 \mathrm{~m}$ bridge

Fig. 7. TLD factors of the three bridge structures with different spans

\subsection{Pavement thickness analysis}

The rigidity coefficients of the assembly hollow slab bridges with spans of $13 \mathrm{~m}$ and $20 \mathrm{~m}$ which are reinforced by different thicknesses $(0,5,10,15,20,25,30,35$, and $40 \mathrm{~cm})$ of overlaid pavement are calculated using the proposed method to analyze the influences of the pavement thickness on the TLD factor. The relationship curves between the pavement thickness and the influence lines of the TLD factors of the bridges with spans of $13 \mathrm{~m}$ and $20 \mathrm{~m}$ and the relationship curves between the pavement thickness and the TLD factors of the bridges with spans of 13, 16, and $20 \mathrm{~m}$ are shown in Fig. 8.

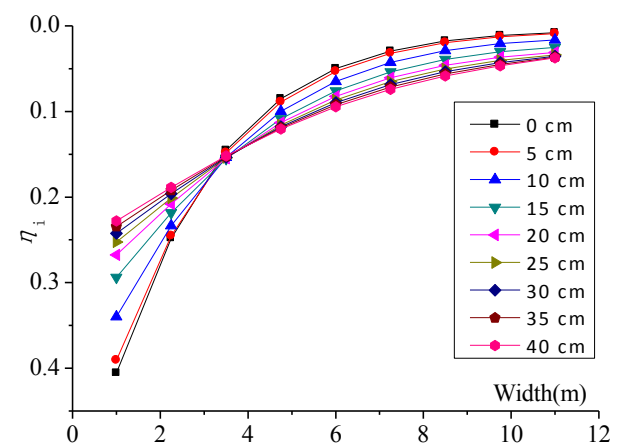

(a) Influence line of the TLD in slab \#1 with $13 \mathrm{~m}$ span



(b) Influence line of the TLD in slab \#5 with $13 \mathrm{~m}$ span



(c) Influence line of the TLD in slab \#1 with $20 \mathrm{~m}$ span

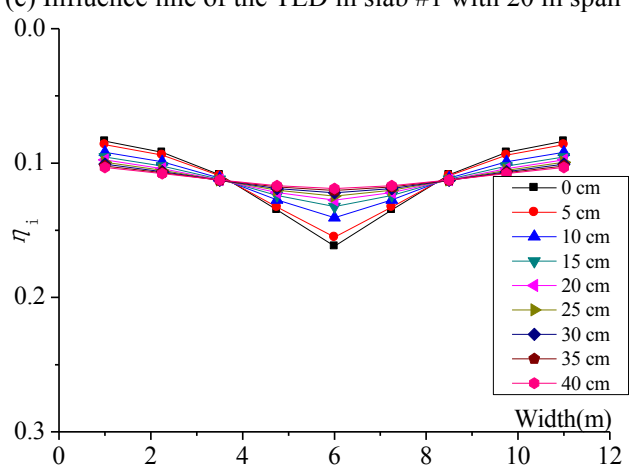

(d) Influence line of the TLD in slab \#5 with $20 \mathrm{~m}$ span



(e) TLD factor of the bridge with $13 \mathrm{~m}$ span

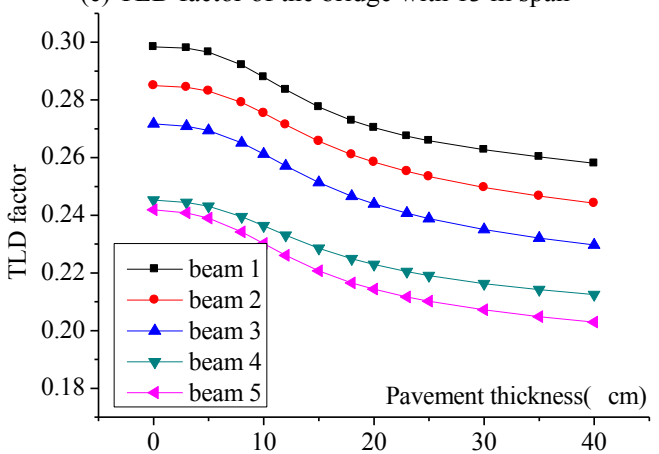

(f) TLD factor of the bridge with $16 \mathrm{~m}$ span

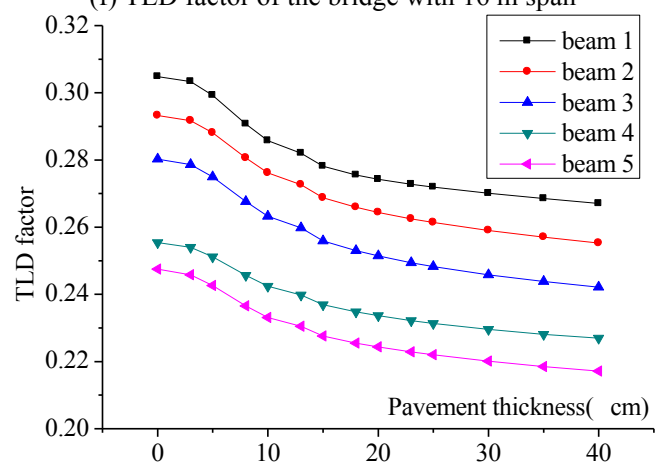

(g) TLD factor of the bridge with $20 \mathrm{~m}$ span

Fig. 8. Effects of pavement thickness on the TLD factor 
Fig. 8. shows that: (1) the influence line of the TLD factor tends to be a straight line as the pavement thickness increases. (2) The influence line of the TLD factor slightly changes after the pavement thickness reaches $20 \mathrm{~cm}$, indicating that the pavement thickness should be smaller than $20 \mathrm{~cm}$. (3) The TLD factor converges gradually with an increase in pavement thickness.

\section{Conclusions}

The mechanical performance of a bridge structure reinforced with overlaid pavement is analyzed in this study to solve the problem of low bearing capacity of assembly slab bridges and increase the utilization of old bridges. Overlaid pavement can increase the structural integrity and improve the load distribution in a girder. Thus, the stresses shared by the overlaid pavement and girder of an assembly slab bridge are analyzed. Furthermore, a calculation model for the assembly slab bridge is established by considering the bending resistance and shearing resistance of the overlaid pavement and using the basic principle of mechanics. The following conclusions are drawn:

(1) The calculation equation for the stresses in the girder is established by considering the bending resistance and shearing resistance of the overlaid pavement. On this basis, the influence line under the effect of the half-sine load is obtained.

(2) The overlaid pavement can significantly influence the vertical coordinate of the influence line. The TLD factor calculated with the bending resistance and shearing resistance of the overlaid pavement considered is smaller than that when the overlaid pavement is disregarded, indicating that the overlaid pavement can enhance the stress uniformity in the girders.
(3) The influences of the overlaid pavement on the calculated results for the assembly slab bridges with small spans $(\leq 16 \mathrm{~m})$ exceed $10 \%$, which should be considered. Nevertheless, the overlaid pavement only minimally influences the calculated results of the assembly slab bridges with large spans $(>16 \mathrm{~m})$, which could be disregarded.

(4) The influence line of the TLD slightly changes after the pavement thickness reaches $20 \mathrm{~cm}$. Thus, the thickness of the reinforced concrete pavement thickness should be controlled within $20 \mathrm{~cm}$.

In this study, a calculation model considering the bending resistance and shearing resistance of the overlaid pavement is established by combining engineering practices and theoretical study. The calculation model can enhance the application of pavement reinforcement to the reconstruction of assembly slab bridges and increase the utilization of old bridges. The overlaid pavement not only enhances the structural integrity and stress uniformity in the girders but also increases the ultimate bearing capacity of the girders. Further research is needed to construct an accurate calculation model for a combined structure composed of pavement and top structure as well as establish a practical algorithm for assembly slab bridges reinforced by overlaid pavement.

\section{Acknowledgments}

This work was supported by the National Basic Research Program of China 973 Plan (project no. 2015CB057701), the Youth Program of the Ministry of Education of Hunan Province (project no. 12B056), and the Science and Technology Planning Project of Changsha (project no. K1309003-11).

This is an Open Access article distributed under the terms of the Creative Commons Attribution Licence

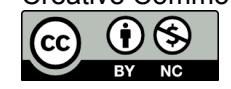

\section{References}

1. GW William, SN Shoukry, MY Riad, "Monitoring of longitudinal cracks in an empirically designed reinforced concrete bridge deck". Bridge Structures, 7(4), 2011, pp.139-149.

2. WJS Gomes, AT Beck, "Optimal inspection and design of onshore pipelines under external corrosion process". Structural Safety, 47(2), 2014, pp.48-58.

3. Jeffrey Luckai, Maria Anna Polak, Scott Walbridge, "A methodology for evaluating the effects of spalling on the structural capacity of reinforced concrete bridge girders". Canadian Journal of Civil Engineering, 41(3), 2014, pp.197-205.

4. G. H. Wang, X. Y. Liu, C. L. Wei, W. W. Wang, L. Shen, and T. X. Jiang, "Incremental Loading Balance Method for the Widening and Reinforcement Design of Old Beam Bridges". Journal of Engineering Science and Technology Review, 9(5), 2016, pp.182 -188.

5. Xiang Yi-Qiang, Xing Cheng, Shao Lin-Hai, ct al., "Spatial Behavior and Strengthening Analysis of Fabricated PC Hollow Slab Beam Bridge with Hinge Joints". Journal of Southeast University ( Natural Science Edition ), 42(4), 2012, pp.734-738.

6. Lin Yang-Zi, Huang Qiao, Ren Yuan, "Reconsidering of Transverse Distribution of Beam-slab Bridge and Problems in Reinforcement". Journal of Highway and Transportation Research and Development, 25(8), 2008, pp.82-86.

7. ZHAN Huaxi, WANG Guoliang, WEI Hongchang, "Systemic Research on the Reinforce Method of Externally-bonded Deck Slab". Journal of Highway and Transportation Research and Development, 23(4), 2006, pp.87-90.

8. WB Stiller, J Gergely, R Rochelle, "Testing, Analysis, and Evaluation of a GFRP Deck on Steel Girders". Journal of Bridge Engineering, 11(4), 2006, pp.394-400.
9. F Natário, MF Ruiz, A Muttoni, "Experimental investigation on fatigue of concrete cantilever bridge deck slabs subjected to concentrated loads". Engineering Structures, 89, 2015, pp.191-203.

10. R Edalatmanesh, JP Newhook, "Behavior of Externally Restrained Noncomposite Concrete Bridge Deck Panels". ACI Structural Journal, 109(2), 2012, pp.161-169.

11. HY Kim, YJ Jeong, "Ultimate strength of a steel-concrete 
composite bridge deck slab with profiled sheeting". Engineering Structures, 32(2), 2010, pp.534-546.

12. M Namy, JP Charron, B Massicotte, "Structural behavior of cast-inplace and precast concrete barriers subjected to transverse static loading and anchored to bridge deck overhangs". Canadian Journal of Civil Engineering , 42(2), 2015, pp.120-129.

13. W Zhang, CS Cai, F Pan, "Nonlinear fatigue damage assessment of existing bridges considering progressively deteriorated road conditions". Engineering Structures, 56(56), 2013, pp.1922-1932.

14. HADIDIR SAADEGHVAZIRIMA, "Transverse Cracking of Concrete Bridge Decks Effects of Design Factors". Journal of Bridge Engineering, 10(5), 2005,pp. 511-519.

15. TW Kim, J Baek, HJ Lee, SY Lee, "Effect of pavement design parameters on the behaviour of orthotropic steel bridge deck pavements under traffic loading". International Journal of Pavement Engineering, 15(5), 2014, pp.471-482.

16. Tang Guo-Bin, Xiang Yi-Qiang, Guan Pin-Wu, "Effect of Deck Pavement on Mechanical Performance of Medium-span and Short-span Bridges". Journal of Highway and Transportation Research and Development, 27(12), 2010, pp.94-98.
17. Lu Da-Wei, Yuan Zhuo-Ya, Shi Xiong-Wei, "Load test efficiency for girder bridges with short or medium span". Journal of Chang'an University (Natural Science Edition), 33(6), 2013, pp.59-67.

18. Liu Hua, Ye Jian-Shu, Yu Bo, "Calculation method of transversal distribution factor for bridge load". Journal of Traffic and Transportation Engineering, 1(9), 2009, pp.62-66.

19. Wang Min-Qiang, Xue Fei, Zhang Heng, "Method of Calculation of Transverse load distribution influence Line for an Articulated Slab Girder Bridge". Engineering Journal of Wuhan University, 37(4), 2001, pp.68-72.

20. Wu Ying-Heng, Zhang Jun-Ping, "Research on force behavior influenced by deck pavement to the simply supported hollow slab". Journal of Guangzhou University (Natural Science Edition), 10(1), 2011, pp. 60-64.

21. Li Guohao, Shi Dong, Calculation of Transverse load distribution For Highway Bridges. 2nd Edition. Beijing: China Communications Press, 1987. 